\title{
First Allowed Bandcrossing in Neutron Deficient Nucleus ${ }^{141} \mathbf{T b}$
}

\author{
N.H. Medina, J.R.B. Oliveira, E.W. Cybulska, M.N. Rao, R.V. Ribas, M.A. Rizzutto, W.A. Seale, \\ Instituto de Física, Universidade de São Paulo, São Paulo, SP, Brazil \\ F.R. Espinoza-Quiñones, \\ Centro de Engenharia e Ciências Exatas, Universidade Estadual do Oeste do Paraná, Toledo, PR, Brazil
}

\author{
D. Bazzacco, F. Brandolini, S. Lunardi, C.M. Petrache, Zs. Podolyák, C. Rossi-Alvarez, \\ F. Soramel, C.A. Ur, \\ Dipartimento di Fisica dell'Università, and INFN, Sezione di Padova, Padova, Italy
}

\author{
M.A. Cardona, G. de Angelis, D.R. Napoli, P. Spolaore, A. Gadea, D. De Acuña, \\ M. De Poli, E. Farnea, D. Foltescu, M. Ionescu-Bujor, and A. Iordachescu \\ INFN, Laboratori Nazionali di Legnaro, Legnaro, Italy
}

Received on 29 October, 2003

\begin{abstract}
The neutron deficient ${ }^{141} \mathrm{~Tb}$ nucleus has been studied with the ${ }^{92} \mathrm{Mo}\left({ }^{54} \mathrm{Fe}, \alpha \mathrm{p}\right)$ reaction at $240-\mathrm{MeV}$ incident energy and the multidetector array GASP. For the yrast $\pi h_{11 / 2}$ decoupled band, excited states up to $6.7 \mathrm{MeV}$ and spin up to $47 / 2^{-}$have been observed. This band presents an upbend at rotational frequency of $\hbar \omega=0.38$ $\mathrm{MeV}$ due to the alignment of $h_{11 / 2}$ protons. The results are discussed in terms of the Cranking model.
\end{abstract}

Neutron-deficient odd-proton nuclei in the A = 140 region display many interesting collective properties. The odd-proton nuclei in this region have moderate prolate quadrupole deformations $\left(\beta_{2} \approx 0.2\right)$ at low spin and are soft with respect to changes in $\gamma$ deformation, which leads to a rich variety of band structures. The $\pi h_{11 / 2}$ orbital plays an important role in the structure of these nuclei. The low- $\Omega$ members of this orbital are close to the Fermi surface, giving rise to decoupled yrast bands at prolate deformations. Pairs of $\pi h_{11 / 2}$ quasiparticles align at modest rotational frequencies stabilizing prolate configurations $\left(\gamma=0^{\circ}\right)$. The alignment of $\nu h_{11 / 2}$ quasiparticles from the upper part of this shell exert a driving force towards collectively rotating oblate nuclear shapes $\left(\gamma=-60^{\circ}\right.$, according to the Lund convention[1]), inducing shape changes in these nuclei.

In order to investigate neutron-deficient nuclei in the $\mathrm{A}=140$ mass region we have performed an experiment with the ${ }^{54} \mathrm{Fe}+{ }^{92} \mathrm{Mo}$ reaction at $240-\mathrm{MeV}$ incident energy. The target used was an approximately $1.0 \mathrm{mg} / \mathrm{cm}^{2}$ thick enriched ${ }^{92}$ Mo foil. The incident beam was obtained with the tandem XTU accelerator of Legnaro National Laboratories, Legnaro, Italy. The multidetector array GASP [2], composed of 40 Compton-suppressed high-efficiency HPGe detectors and the 80-element BGO inner ball, was used for obtaining gamma-ray double and triple coincidence spectra. The multi-telescope light-charged-particle detector array (ISIS) [3], consisting of $40 \mathrm{Si}$ surface-barrier $\Delta \mathrm{E}-\mathrm{E}$ telescopes, enabled the selection of the multiplicities of the evaporated charged particles in coincidence with the observed $\gamma$-rays. In order to permit the identification of the various masses produced in the reaction, the recoil mass spectrometer CAMEL [4] was coupled to GASP. The coupled GASP-CAMEL system has an intrinsic mass resolution better than 1/300 and the transmission efficiency in this experiment was about $1 \%$. Events have been collected on tape when at least two Compton-suppressed HPGe detectors and two BGO detectors fired in coincidence. A total of $1.3 \times 10^{9}$ Compton-suppressed events was collected. The data have been Doppler corrected and sorted into charged particle- $\gamma$ $\gamma$, mass $-\gamma-\gamma$ and $\gamma-\gamma-\gamma$ cubes. Various nuclei $(Z=63$ to 66 ; $\mathrm{N}=75$ to 78 ) were produced in this experiment, the $3 \mathrm{p}$ channel leading to ${ }^{143} \mathrm{~Tb}$ was the most strongly populated nucleus and the results on the high-spin structures observed were described in Ref.[5], where a more detailed description of the experiment and the data analysis may be found. Other nuclei studied in this experiment were ${ }^{140} \mathrm{~Tb}$ [6], and ${ }^{143}$ Dy [7]. We report here the results on the $\alpha p$ channel leading to the neutron deficient nucleus ${ }^{141} \mathrm{~Tb}$. This nucleus is the heaviest $\mathrm{N}=76$ odd-proton nucleus to be studied at high spin by means of $\gamma$-ray spectroscopy. The ground state of this nucleus is known to be $5 / 2^{-}[8]$, and previous to the present work only the first three low-lying $\gamma$-transitions emitted from the levels populated with the heavy ion reaction ${ }^{54} \mathrm{Fe}+{ }^{92} \mathrm{Mo}$ at $260 \mathrm{MeV}$ were known: 307.3, 503.8 and $647.5 \mathrm{keV}$ [9].

The comparison with the mass-141 gated spectra and the particle gated spectra permitted unambiguous assignment of newly observed $\gamma$-transitions to the ${ }^{141} \mathrm{~Tb}$ nucleus. Background-subtracted spectra generated from those matrices were used to construct the level scheme. Those matrices 
were analysed using the VPAK [10] and RADWARE [11] spectrum analysis codes. The fully symmetrized $\gamma-\gamma-\gamma$ cube was used to construct $\gamma-\gamma$ matrices in coincidence with several transitions, confirming and extending the level scheme of ${ }^{141} \mathrm{~Tb}$ (Fig. 1). The assignment of the spins and parities to the ${ }^{141} \mathrm{~Tb}$ levels was based on the DCO (directional correlation from oriented states) ratios [12].

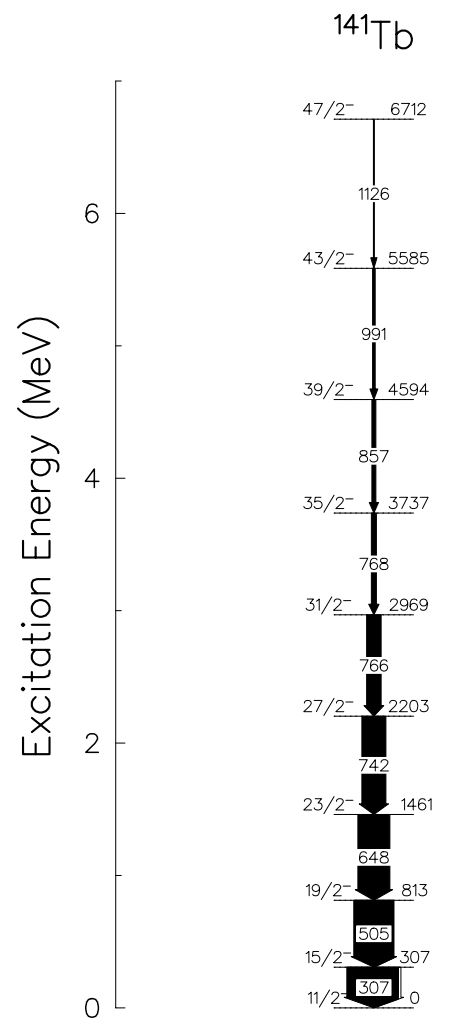

Figure 1. The level scheme of ${ }^{141} \mathrm{~Tb}$ obtained from the present work.

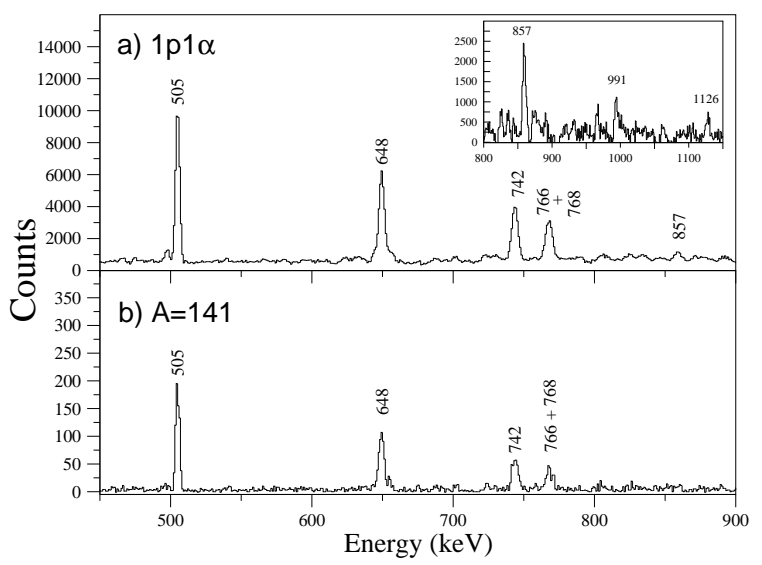

Figure 2. Gamma-ray spectra in coincidence with the $307-\mathrm{keV}$ line. a) gated with particle fold $1 \mathrm{p} 1 \alpha$. In the inset high-energy transitions connecting the high-spin states are shown. b) gated on mass $A=141$. The peaks present in both spectra can be assigned to ${ }^{141} \mathrm{~Tb}$.
In Fig. 2 the $1 \alpha 1$ p particle-gated (a) and mass-141 gated (b) spectra in coincidence with the $307 \mathrm{keV}$ transition are shown. In the inset the high-energy transitions connecting the high-spin states are shown. The peaks present in both spectra are assigned to ${ }^{141} \mathrm{~Tb}$.

Several sequences of $\gamma$-rays could be assigned to the ${ }^{141} \mathrm{~Tb}$ nucleus based on A-gated and charge-particle multiplicity gate, but the placement of theses sequences on the level scheme was not possible.

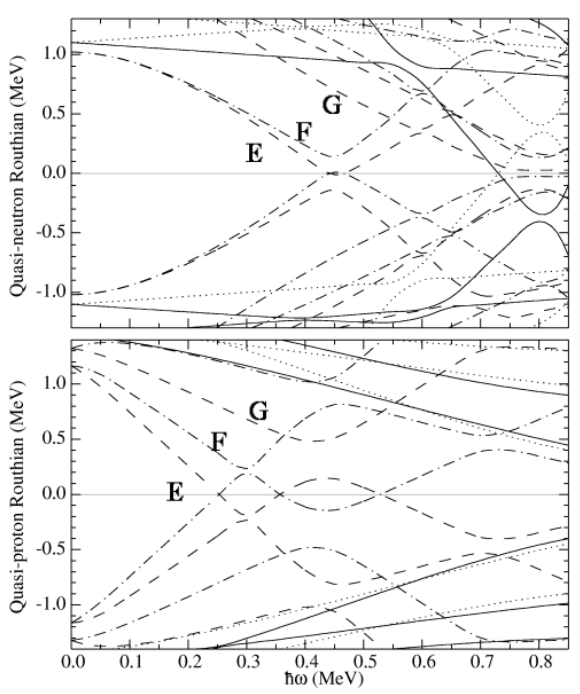

Figure 3. Quasiparticle Routhians as a function of rotational frequency, assuming deformation parameters of: $\beta_{2}=0.229, \beta_{4}=$ -0.033 and $\gamma=-22.6^{\circ}$. Top: quasineutrons; bottom: quasiprotons.

The angular momentum of the yrast band observed in ${ }^{141} \mathrm{~Tb}$ presents a sharp upbend at a rotational frequency of $\hbar \omega=0.38 \mathrm{MeV}$ (Fig. 4). The gain in alignment at that frequency is about $4.5 \hbar$. The TRS [13] calculations predict quasiparticle alignments of both protons and neutrons around that frequency with a very large gain in total alignment (about 12 $\hbar$ ). It is more likely, however, that only the alignment of protons is observed experimentally. CSM calculations (Fig. 3) predict a gain of 5.6 $\hbar$ in alignment from the first allowed $\pi h_{11 / 2}$ crossing (FG, at $\hbar \omega=0.42 \mathrm{MeV}$ ), and $6.9 \hbar$ for the $\nu h_{11 / 2}$ EF crossing (at $\hbar \omega=0.46 \mathrm{MeV}$ ), assuming deformation parameters of: $\beta_{2}=0.229, \beta_{4}=$ -0.033 and $\gamma=-22.6^{\circ}$ (from the TRS minimum obtained for the E quasiproton configuration at $\hbar \omega=0.298 \mathrm{MeV}$ ). In order to obtain the TRS predictions for the three-quasiproton configuration (EFG) at high frequency, we performed the calculations with the excitation of a pair of negative parity quasineutrons. Above the quasineutron crossing frequency this corresponds to the diabatic continuation of the neutron quasiparticle vacuum. In this manner we followed the sequence of calculated quantities which correspond to the crossing of the E with the EFG quasiproton configurations, while the neutrons remain in the quasiparticle vacuum. In other words, below the crossing frequency, we followed the 
lowest negative parity excitation, while above it, the second lowest. The total spin as a function of rotational frequency calculated by the TRS code with this procedure is presented in Fig. 4 together with the experimental values. The agreement between the two is quite reasonable; the calculations, however, overestimated the alignment spin gain in the crossing by about $2 \hbar$. Similar results were obtained for the isotone ${ }^{139} \mathrm{Eu}[14]$. It should be pointed out that, in this figure the choice of the calculated points nearby the crossing frequency (which link between the two crossing configurations) is rather arbitrary, and was guided by the experimental results. The origin of this dificulty is related to the use of a frequency (which is the free parameter) step of calculated data points, rather than a spin step (which, incidentally, should be of two units, corresponding to E2 transitions). On the other hand, sufficiently far from the crossing frequency no ambiguity remains and the choices are independent of the experiment.

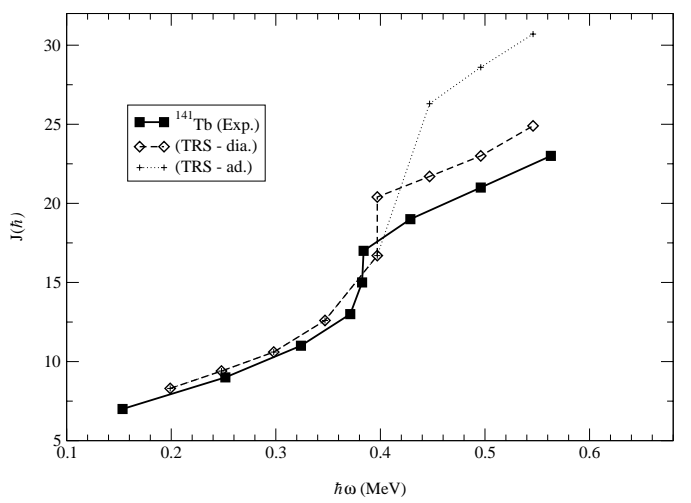

Figure 4. Angular momentum as a function of rotational frequency. The closed circles linked by solid lines are experimental data. The crosses linked by dotted lines are adiabatic TRS calculations, while open diamonds linked by dashed lines are diabatic TRS calculations.

The TRS calculations, assuming the absolute minimum reproduce quite well the experimental trends and crossing frequencies including the gradual increase of the alignment in ${ }^{141} \mathrm{~Tb}$ below the crossing, which is a contrasting feature in comparison to the nearby isotones. Therefore, the assumption that the observed band is the continuation of the EFG quasiproton configuration above the neutron crossing frequency is reasonable for ${ }^{141} \mathrm{~Tb}$. The absence of the neutronaligned pair coupled to EFG quasiproton band in the experiment could, hypothetically, be related to the formation of an isomeric bandhead state. The identification of the band in this case would be very difficult since only a thin target experiment was analised. A few nanosseconds are sufficient for the recoiling nuclei to fly away from the focus of the spectrometer, and the connection to lower lying states would not be observed.
In conclusion the high spin states of the neutron deficient ${ }^{141} \mathrm{~Tb}$ nucleus were measured for the first time. For the yrast $\pi \mathrm{h}_{11 / 2}$ decoupled band, excited states up to 6.7 $\mathrm{MeV}$ and spin up to $47 / 2^{-}$have been observed. This band presents an upbend at rotational frequency of $\hbar \omega=0.38$ $\mathrm{MeV}$ due to the alignment of $\mathrm{h}_{11 / 2}$ protons. The results for the yrast band add important information with regards to the systematics of bandcrossings towards the most neutron deficient nuclei in the mass-140 region, and to the proof of theoretical model calculations.

We thank Mr. G Manente for the preparation of the targets and the staff of the XTU-Tandem of LNL for the smooth operation of the accelerator. This work was partially supported by Istituto Nazionale di Fisica Nucleare (INFN), Italy, Fundação de Amparo à Pesquisa do Estado de São Paulo (FAPESP) and Conselho Nacional de Desenvolvimento Científico e Tecnológico (CNPq), Brazil.

\section{References}

[1] G. Andersson, S. E. Larsson, G. Leander, P. Möller, S. G. Nilsson, I. Ragnarsson, S. Aberg, R. Bengtsson, J. Dudek, B. Nerlo-Pomorska, K. Pomorski, and Z. Szymański, Nucl. Phys. A 268, 205 (1976)

[2] D. Bazzacco, in Proceedings of the International Conference on Nuclear Structure at High Angular Momentum, Ottawa (1992) Report No. AECL 10613, Vol. 2, p. 376.

[3] E. Farnea et al., Nucl. Instr. Meth. A 400, 87 (1998).

[4] P. Spolaore, J. D. Larson, C. Signorini, S. Beghini, Z. Xi-Kai, and S. Hou-Zhi, Nucl. Instr. Meth. A 238, 381 (1985).

[5] F.R. Espinoza-Quiñones et al., Phys. Rev. C 60, 054304 (1999).

[6] M.A. Rizzuto et al., Phys. Rev. C 62, 027302 (2000).

[7] J.R.B. Oliveira et al., Phys. Rev. C 62, 064301 (2000).

[8] J.K. Tuli and D.F. Winchell, Nucl. Data Sheets 92, 277 (2001).

[9] L. Goetting et al., Nucl. Phys. A 745, 569 (1987).

[10] W. T. Milner, Holifield Heavy Ion Research Facility Computer Handbook, Oak Ridge National Laboratory, Oak Ridge, Tennessee, USA (1987).

[11] D. Radford, Nuclear Inst. Meth. A 361, 297 (1995).

[12] J.E. Draper, Nuclear Inst. Meth. A 247, 418 (1986).

[13] R. Wyss, J. Nyberg, A. Johnson, R. Bengtsson, and W. Nazarewicz, Phys. Lett. B 215, 211 (1988).

[14] P. Vaska, C.W. Beausang, D.B. Fossan, J.R. Hughes, R. Ma, E.S. Paul, R.J. Poynter, P.H. Regan, R. Wadsworth, S.A. Forbes, S.M. Mullins and P.J. Nolan, Phys. Rev. C 52, 1270 (1995). 